\title{
QUERATOSIS SEBORREICA DE LA CONJUNTIVA: INFORME DE UN CASO
}

\section{SEBORRHEIC KERATOSIS OF CONJUNCTIVA: A CASE REPORT}

\author{
GULIAS-CAÑIZO R ${ }^{1}$, ARANDA-RÁBAGO J ${ }^{2}$, RODRÍGUEZ-REYES AA ${ }^{3}$
}

\begin{abstract}
RESUMEN
Caso clínico: Las lesiones conjuntivales pigmentadas representan un reto diagnóstico para el clínico. Hombre de 37 años de edad quien presentó una masa conjuntival pigmentada en su ojo derecho. Clínicamente se diagnosticó como melanoma, por lo que se realizó escisión completa del tumor. El diagnóstico histopatológico fue de queratosis seborreica.

Discusión: La queratosis seborreica es una lesión benigna que se presenta en los párpados y en la cara de individuos de edad media y adultos mayores. El desarrollo de esta lesión en la conjuntiva es poco frecuente, solo se han informado 2 casos en la literatura. La queratosis seborreica debe ser considerada en el diagnóstico diferencial de lesiones conjuntivales pigmentadas.
\end{abstract}

Palabras clave: Conjuntiva, queratosis seborreica, tumores conjuntivales pigmentados, melanoma conjuntival, queratosis folicular invertida.

\begin{abstract}
Case report: Pigmented conjunctival lesions are a diagnostic challenge for the clinician. A 37-yearold man presented with a pigmented mass involving the conjunctiva of his right eye. Clinically, a diagnosis of malignant melanoma was made and a wide excision of the tumor was performed. The histopathologic diagnosis was seborrheic keratosis.

Discussion: Seborrheic keratosis is a benign lesion which occurs on the eyelids and face of middleaged and elderly individuals. The occurrence of this lesion on the conjunctiva is rare, however there are 2 cases reported in the world literature. Seborrheic keratosis should be considered in the differential diagnosis of conjunctival pigmented lesions (Arch Soc Esp Oftalmol 2006; 81: 217-220).
\end{abstract}

Key words: Conjunctiva, seborrheic keratosis, conjunctival pigmented tumors, conjunctival melanoma, inverted follicular keratosis.

Recibido: 28/9/05. Aceptado: 11/4/06

Asociación para Evitar la Ceguera en México, I.A.P., Hospital «Dr. Luis Sánchez Bulnes».

1 Doctor en Medicina. Oftalmólogo.

2 Doctor en Medicina. Oftalmólogo-Segmentólogo.

3 Doctor en Medicina. Anatomopatólogo. Patólogo Oftálmico.

Correspondencia:

Rosario Gulias Cañizo

Asociación para Evitar la Ceguera en México, I.A.P.

Hospital «Dr. Luis Sánchez Bulnes»

Vicente García Torres \# 46, Col. San Lucas Coyoacán,

04030 México, D.F

México

E-mail: bichorro76@hotmail.com 


\section{INTRODUCCIÓN}

Las lesiones conjuntivales pigmentadas representan un reto diagnóstico para el clínico. En algunos casos, lesiones de curso aparentemente indolente representan diagnósticos con un mal pronóstico. En otros casos, tumoraciones de crecimiento rápido que sugieren neoplasias malignas en realidad son lesiones benignas con buen pronóstico. Por lo anterior, es de suma importancia estar familiarizado con el aspecto clínico y el diagnóstico histológico de las distintas entidades conjuntivales que pueden producir confusión en el momento del diagnóstico y provocar un tratamiento inadecuado.

\section{CASO CLÍNICO}

Hombre de 37 años de edad sin antecedentes de importancia quien desarrolló una masa conjuntival en el ojo derecho (OD) de 8 meses de evolución. Clínicamente se encontró una masa pigmentada sobre la conjuntiva nasal que invadía parte de la córnea pero no afectaba el eje visual. A la exploración oftalmológica inicial, tenía una agudeza visual de 20/20 y una presión intraocular de $12 \mathrm{mmHg}$ en ambos ojos. Tanto la biomicroscopía como la oftalmoscopía indirecta del ojo izquierdo (OI) fueron normales. La biomicroscopía del OD reveló una masa densamente pigmentada, elevada, de 9 × 4 $\mathrm{mm}$., de bordes bien delimitados, localizada en el sector nasal de la conjuntiva bulbar y que invadía parte de la córnea (fig. 1). La masa se encontraba

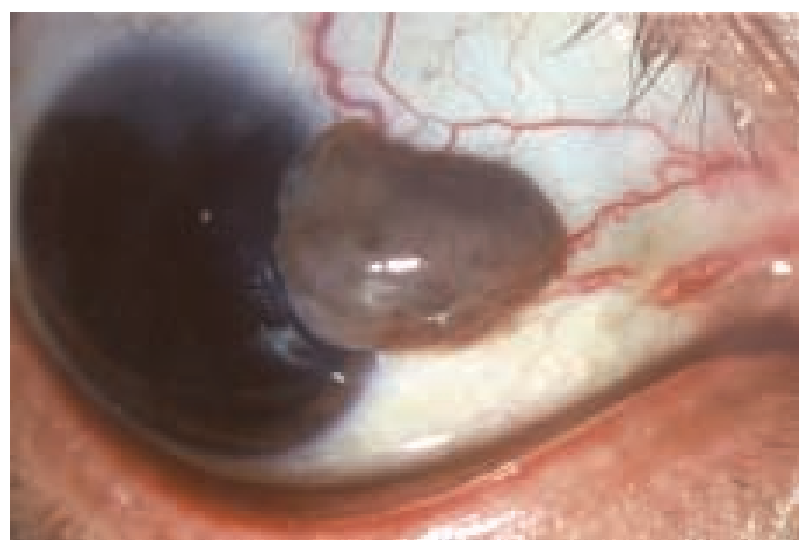

Fig. 1: Aspecto clínico que muestra lesión pigmentada paralímbica de bordes bien definidos con extensión a la córnea. rodeada por algunos vasos nutricios prominentes. No hubo evidencia de melanosis adquirida primaria adyacente a la lesión. El resto de la exploración oftalmológica, incluyendo la fundoscopía, resultó normal.

Se realizó una escisión completa de la lesión con márgenes quirúrgicos amplios. El examen macroscópico reveló una lesión con dimensiones de 8 x 4 x $3 \mathrm{~mm}$, de forma nodular, superficie externa lisa, multilobulada, de color café negruzco y aspecto mucoso.

El examen histopatológico demostró epitelio de revestimiento conjuntival con cambios de papilomatosis y acantosis irregular, esta última a expensas de proliferación de células basaloides, mismas que estaban mezcladas con pequeños grupos de células escamosas y algunos melanocitos dendríticos (fig. 2). La sustancia propia presentaba además, áreas con degeneración elastótica, vasos sanguíneos de pequeño calibre dilatados y cúmulos variables de infiltrado inflamatorio formados por linfocitos maduros, células plasmáticas y algunos melanófagos.

Con estos hallazgos se estableció el diagnóstico de queratosis seborreica (QS) de la conjuntiva.

\section{DISCUSIÓN}

La QS es una neoplasia epitelial benigna que se presenta con cierta frecuencia en la piel de los párpados y en la cara de individuos entre la cuarta y quinta décadas de la vida (1). En la conjuntiva, presenta un crecimiento relativamente rápido, así como

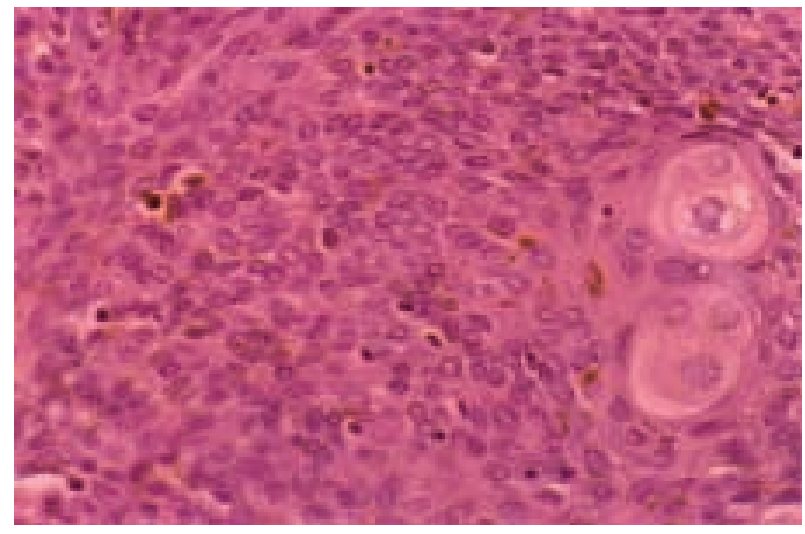

Fig. 2: Fotomicrografía que muestra proliferación de células basaloides mezcladas con pequeños nidos de células escamosas y melanocitos dendríticos ( $H-E$, aumento original $\times 20$ ). 
frecuentemente la presencia de pigmento melánico tanto clínica como histológicamente (4), lo cual invita a pensar en una neoplasia maligna del tipo del melanoma. Por tal motivo, se recomienda realizar toma de biopsia o resección completa de la lesión con su respectivo estudio histopatológico, para así poder establecer el diagnóstico definitivo.

Hasta el momento actual, solo se han informado dos casos en la literatura de esta entidad con afección conjuntival, uno de ellos diagnosticado como QS y el otro como una variante de la misma denominada queratosis folicular invertida (QFI) $(2,3)$.

Los dos casos correspondieron a pacientes del sexo masculino de 66 y 21 años de edad respectivamente, sin antecedentes de importancia, quienes presentaron una masa conjuntival de crecimiento rápido. Las lesiones midieron 4 y $8 \mathrm{~mm}$ de eje mayor respectivamente; la primera era densamente pigmentada y la segunda de color amarillento. Ambas lesiones se localizaban en la zona interpalpebral paralímbica. En el primer caso se hizo el diagnóstico clínico de melanoma maligno.

En los dos pacientes se realizó escisión completa de la lesión. Los hallazgos histopatológicos fueron similares en los dos casos, caracterizados por la presencia de epitelio acantósico a expensas de proliferación de células basaloides, sin atipia celular, pleomorfismo nuclear ni mitosis. En el primer caso se observaba además queratinización leve, incremento de la pigmentación del epitelio conjuntival, así como la presencia de algunos quistes córneos. En el segundo, las células basaloides acantolíticas estaban dispuestas alrededor de «remolinos escamosos», por lo que se diagnosticó como QFI.

Nuestro caso comparte muchas características con los dos previamente informados, ya que también se trató de un paciente del sexo masculino, de 37 años de edad, sin antecedentes de importancia y con una lesión conjuntival pigmentada de crecimiento rápido en la misma localización. El diagnóstico clínico en nuestro paciente fue de melanoma conjuntival probablemente «de novo», por lo que fue tratado mediante escisión completa de la lesión. Los hallazgos histopatológicos fueron casi indistinguibles a los informados por Tseng et al (2), con la excepción de que en nuestro caso no encontramos la formación de quistes córneos. Es importante mencionar que tanto en los dos casos previamente informados en la literatura $(2,3)$, como en nuestro caso, el epitelio de revestimiento conjuntival afectado no demostró remanentes de células productoras de moco o caliciformes, ni cambios de displasia.

Ante la presencia de una neoplasia conjuntival pigmentada, es obligado realizar una escisión amplia y completa de la lesión ya que si existe la sospecha de melanoma, aunque raro en esta localización, su mortalidad es de aproximadamente del 30 al $40 \%$ (4).

La QS como su variedad QFI de la conjuntiva, presentan hallazgos histopatológicos muy similares a aquellas que se originan en la piel. Vale la pena enfatizar que cuando alguna de ellas se desarrolla en la conjuntiva, generalmente son pigmentadas (5), por lo que deben considerarse en el diagnóstico diferencial junto con el melanoma conjuntival.

\section{BIBLIOGRAFÍA}

1. Albert DM, Jackobiec FA. Principles and Practice of Ophthalmology: Clinical Practice. Vol. 3. Philadelphia: Saunders; 1994; 1716-1717.

2. Tseng SH, Chen YT, Huang FC, Jin YT. Seborrheic keratosis of conjunctiva simulating a malignant melanoma: an immunocytochemical study with impression cytology. Ophthalmology 1999; 106: 1516-1520.

3. Cakmak SS, Unlu MK, Bilek B, Buyukbayram H, Sakalar $Y B$. Conjunctival inverted follicular keratosis: a case report. Jpn J Ophthalmol 2004; 48: 497-498.

4. Rodríguez Reyes AA. Fascículos de Enseñanza Clinicopatológica: Conjuntiva y carúncula. $1 .^{a}$ ed. No. 2. México: Publicaciones Oftalmológicas de la Asociación para Evitar la Ceguera en México, I.A.P. Hospital «Dr. Luis Sánchez Bulnes». 2001; 7.

5. Seregard S. Conjunctival melanoma. Surv Ophthalmol 1998; 42: 321-350. 